\title{
Producing single-photon wave packets of the infrared spectrum range with an orbital angular momentum using "vortex" phase plates
}

\author{
D.A. Turaykhanov ${ }^{1}$, A.V. Shkalikov ${ }^{1, *}$, A.A. Kalachev ${ }^{1}$, I.R. Imangulova ${ }^{2}$, N.N. Losevsky ${ }^{2}$, \\ E.V. Razova ${ }^{2}$, S.A. Samagin², S.P. Kotova ${ }^{2}$ \\ ${ }^{1}$ Zavoisky Physical-Technical Institute, Kazan Scientific Center RAS, 420029 Kazan, Russia \\ ${ }^{2}$ P.N. Lebedev Physical Institute RAS, Samara Branch, 443011 Samara, Russia
}

\begin{abstract}
We consider the peculiarities of formation and registration of axially symmetric vortex fields in the context of applying diffractive optical elements such as vortex lenses and vortex axicons for the generation of single-photon states with a nonzero orbital angular momentum in the process of spontaneous parametric down-conversion.
\end{abstract}

For the realization of free-space quantum communication channels, light beams with an orbital angular momentum (OAM) are of greatest interest [1,2]. Experimental and theoretical studies show that such beams are more stable when propagating in a turbulent medium compared to ordinary Gaussian beams [3, 4], and exhibit self-healing ability [5, 6].

Single-photon states at a wavelength around $800 \mathrm{~nm}$ corresponding to one of the atmospheric transparency windows can be effectively obtained in the process of spontaneous parametric down-conversion (SPDC) of light in a nonlinear medium [7]. In this paper, we present the results of preliminary studies devoted to generation of single-photon states with a nonzero OAM in the process of SPDC with the use of diffractive optical elements.

To create optical vortices we used diffractive optical elements (DOE) in the form of a vortex lens and a vortex axicon $[8,9]$, which is one of the most flexible and energetically effective methods. We studied theoretically and experimentally the evolution of the generated fields during their propagation near the focusing region for a vortex lens and a vortex axicon. Fig. 1 shows the diameter of the beam ring as a function of the distance along the propagation axis. It can be seen that for the axicon the distance along the propagation axis, over which the intensity distribution remains practically constant, is about 2 times larger than that for the vortex lens. For the mask parameters used in the experiment, it is about $4 \mathrm{~cm}$ and $2 \mathrm{~cm}$, respectively. On the other hand, the vortex lens provides a greater contrast of intensity distribution in the focal plane, which can be important when detecting the output signal. Therefore, when creating an experimental setup, the problem of choosing between vortex lenses and vortex axicons should be solved taking into account the geometry of the pump field and the geometry of the registration scheme.

\footnotetext{
* Corresponding author: andrei_vs@ rambler.ru
} 


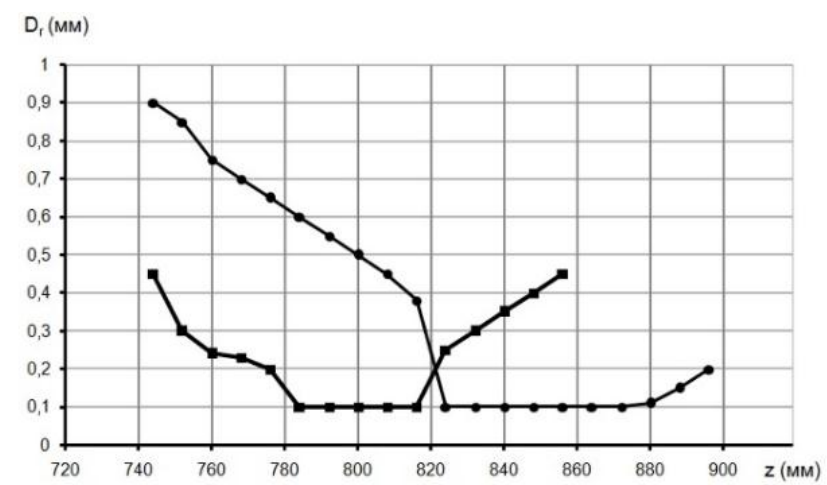

Fig. 1. The width of the beam ring as a function of the distance in the focus region for the axicon (circles) and for the vortex lens (squares).

One of the most effective methods for generating single photons is the process of SPDC, during which a photon from a strong pump field propagating in a non-centrosymmetric medium is annihilated with creating two photons, called idler and signal. This process satisfies the energy and phase matching conditions: $\boldsymbol{\omega}_{\boldsymbol{p}}=\boldsymbol{\omega}_{\boldsymbol{i}}+\boldsymbol{\omega}_{\boldsymbol{s}}$ and $\overrightarrow{\mathrm{k}}_{\boldsymbol{p}}=\overrightarrow{\mathrm{k}}_{\boldsymbol{i}}+\overrightarrow{\mathrm{k}}_{\boldsymbol{s}}$, respectively, where $\omega$ and $\overrightarrow{\mathrm{k}}$ are the frequency and wave vector, respectively, of the signal $(i)$, idler $(s)$ and pump $(p)$ photons. In addition, for the collinear SPDC the following condition is valid for OAM: $\ell_{\boldsymbol{p}}=\ell_{\boldsymbol{s}}+\ell_{\boldsymbol{i}}$. We numerically simulated the collinear SPDC process for $\ell_{\boldsymbol{p}}=\mathbf{- 1}, \mathbf{0},+\mathbf{1},+\mathbf{2}$, and calculated coincidences between the signal and idler photons for 49 possible combinations of orthogonal states. As should be expected, the coincidences observed in all cases when the sum of OAM of the generated photons is equal to that of the pump beam.

The paper presents the results of experimental studies devoted to the generation of single photons with a non-zero OAM via SPDC. We consider the peculiarities of formation and registration of axially symmetric vortex fields with the use of diffractive optical elements: vortex lenses and vortex axioms. It was found that the position of the detection plane in the case of vortex lenses is more critical than in the case of vortex axioms. Compensatory method of measuring the topological charge allows one to achieve at least a tenfold increase in the signal compared to the method without compensation.

This work was supported by the Russian Foundation for Basic Research (project No. 16-02-01055-a) and by the theme of the state assignment No. 0217-2018-0005 (numerical simulations of the SPDC process).

\section{References}

1. A.M. Yao, M.J. Padgett, Adv. Opt. Photon., 3, 161 (2011)

2. J. Wang, Photon. Res., 4, B14 (2016)

3. C. Paterson, Phys. Rev. Lett, 94, 153901 (2005)

4. A. Vinotte and L. Berg, Phys. Rev. Lett, 95,193901 (2005)

5. Z. Bouchal, Opt. Commun, 210, 155 (2002)

6. M. V. Vasnetsov et al., JETP Lett, 71, 130 (2000)

7. D.N. Klyshko, Photons and Nonlinear Optics, Gordon \& Breach (1988)

8. E. Abramochkin et al., Optics Comm, 102, 336 (1993)

9. A.V. Shkalikov et al., Bull. Lebedev Phys. Inst., 45, 79 (2018) 